\title{
Information Theoretical Estimators of Tuning Depth and Time Delay for Motor Cortex Neurons
}

\author{
Yiwen Wang, Student Member, Justin C. Sanchez, Member, Jose C. Principe, Fellow
}

\begin{abstract}
Knowledge of neural receptive fields helps decode animal's movement from neural activity in motor brain machine interfaces (BMI). The traditional tuning depth is a coarse metric to evaluate the neuron's tuning properties. The major difficulty of the tuning depth is that it does not allow for a direct comparison among neurons that have very different firing rates. An information theoretic tuning depth based on mutual information is proposed to evaluate how neural spikes encode the kinematic direction. This metric is scale invariant and makes the tuning analysis comparable among not only the cortical areas but also among kinematics vectors. It can help identify candidate neuron subsets to reduce the complexity of analysis and decoding in BMI. The application of the metric on neural encoding modeling also provides a way to estimate time delays in motor cortical neural activity.
\end{abstract}

\section{INTRODUCTION}

The neural representation of information requires an understanding of three interconnected aspects: 1) What is the stimulus? 2) How does the neuron encode it? 3) What is the criterion for quantifying the quality of the response? The tuning curve was proposed initially as a cosine curve between the stimulus and the response [1] because of the broad representation observed in experimental paradigms.

For neurons located in the motor cortex, significant evidence shows that neurons are sensitive to the movement direction and velocity [2], [3], [4]. To quantify the degree of tuning, a metric of the tuning depth has been proposed. This quantity is defined as the difference between the maximum and minimum values in the firing rates, normalized by the standard deviation of the firing rate [5] and is a good indicator of neuronal involvement in a task. As a scalar, the tuning depth summarizes the statistical information contained in the tuning curve to evaluate the receptive fields properties of the neurons. However, this metric has some shortcomings in implementation. It can exaggerate the value of tuning depth when the neuron firing rate standard deviation is close to 0 . Additionally, it depends on the binning window size to calculate the firing rate of the neuron. The tuning depth also relates to the scale of the stimulus and makes the analysis not comparable among neurons as we will see. A more principled metric, allowing comparisons among neurons and among

This work was supported by National Science Foundation under grant no CISE-0541241, ECS-0422718, CNS-0540304 and the Children's Miracle Network.

Y. Wang and J.C. Principe are with the Computational NeuroEngineering Laboratory at the Department of Electrical and Computer Engineering, University of Florida, Gainesville, FL 32611 (e-mail: \{wangyw, principe\}@cnel.ufl.edu).

J.C. Sanchez is with the Neuroprosthesis Research Group at the Department of Pediatrics, University of Florida, Gainesville, FL 32610 (email: jcs 77 @ufl.edu) kinematic variables, is necessary to mathematically evaluate the information encoded by neurons about the kinematics variables. When this is achieved, the new tuning depth metric can be utilized to select neural subsets that contribute the most to movement generation, potentially reducing the complexity of the BMI model.

In addition to tuning depth, researchers have also proposed different simple parametric models to describe the stimulusresponse functional properties of motor cortex neurons, such as linear tuning models [6], [7], Gaussian tuning for hippocampal pyramidal neurons [8], and a projective nonlinearPoisson model [9] for visual system based on a white noise analysis [10]. The modeling analysis provides a deeper understanding of how neuron spikes respond to a stimulus, and holds promise toward providing analytical solutions to the underlying mechanism of neuron receptive fields. However, the analysis of the receptive fields of motor cortex neurons is different from the stimulus-response analysis in sensory cortices because of the long delays. Indeed, in the primary motor cortex neurons there is always a time delay between the initiation of the action and the response. When the goal is to model the instantaneous kinematics as the state in generative BMI modeling, we have to deal with this delay.

This paper proposes the information theoretical concept of mutual information to estimate tuning depth to analyze the information that neurons in different cortical areas share from the directions of animal's position, velocity and acceleration. The metric is also applied in section II to the projective nonlinear-Poisson encoding analysis to estimate the best time delay in motor responses to model motor cortical neurons.

\section{DATA COLLECTION AND METHODS}

\section{A. Data collection}

The Brain Machine Interfaces paradigm was designed and implemented by Nicolelis lab at Duke University. The electrical neural activities were recorded invasively in the brain of an adult female Rhesus monkey, and synchronized with task behaviors. Several micro-electrode arrays were chronically implanted in five of the monkey's cortical neural structures: right dorsolateral premotor area $(\mathrm{PMd})$, right primary motor cortex (MI), right primary somatosensory cortex (S1), right supplementary motor area (SMA), and the left primary motor cortex (MI). Each electrode array consisted of up to 128 microwires (30 to $50 \mu \mathrm{m}$ in diameter, spaced $300 \mu \mathrm{m}$ apart), distributed in a 168 matrix. Each recording site occupied a total area of $15.7 \mathrm{~mm}^{2}(5.6 \times 2.8 \mathrm{~mm})$ and was capable of recording up to four single cells from each microwire for a total of 512 neurons (4128) [5]. After the surgical procedure, 
a multi-channel acquisition processor (MAP, Plexon, Dallas, TX) cluster was used in the experiments to record the neuronal action potentials simultaneously. Analog waveforms of the action potential were amplified and band pass filtered from $500 \mathrm{~Hz}$ to $5 \mathrm{kHz}$. The spikes of single neurons from each microwire were discriminated based on time-amplitude discriminators and a principal component (PC) algorithm [11], [12]. The firing times of each spike were stored. Table 3-1 shows the assignment of the sorted neural activity to the electrodes for different motor cortical areas [13]. The monkey performed a two-dimensional target-reaching task to move the cursor on a computer screen by controlling a handheld manipulandum in order to reach the target. The monkey was rewarded when the cursor intersected the target. The corresponding position of the manipulandum was recorded continuously for an initial $30-$ min period at a $50 \mathrm{~Hz}$ sampling rate, referred to as the "pole control" period [14].

\section{B. Tuning Depth Analysis}

Neurophysiologic evidence suggests that the direction of hand movements is encoded in cells with cosine shaped tuning curves. For each neuron, the polar plot of the neuron activities with regard to a kinematic vector, such as hand position, hand velocity, or hand acceleration, is investigated to compute the kinematic direction as an angle between 0 and 360 degrees. 45 degree bins are then chosen to coarsely classify all the directions into 8 bins. For each direction, the average firing rate for a neuron by window-binning is computed as the magnitude of the vector in the polar plot. For a tuned neuron, the average firing rates for each direction are expected to be quite different. The preferred direction is computed using circular statistics [15] as shown in (1).

$$
\text { circular mean }=\arg \left(\sum_{N} r_{N} e^{i \theta_{N}}\right)
$$

where is the neuron's average firing rate for angle, and $\mathrm{N}$ covers all the angle range. The metric for evaluating the tuning property of a cell is the tuning depth. This quantity is defined as the difference between the maximum and minimum values in the cellular tuning normalized by the standard deviation of the firing rate [5]. The tuning depth is normalized between 0 and 1, which looses the scale when compared against different neurons.

$$
\text { tuning depth } \left.=\frac{\max \left(r_{N}\right)-\min \left(r_{N}\right)}{\text { std }(\text { firingrate })}\right)
$$

The normalization techniques used to equalize the firing of different cells can wrongly evaluate a shallow tuned neuron with a deeply tuned neuron, when both fire with a small variance. The normalization in the tuning depth inaccurately exaggerates the tuning depth when the standard deviation is close to 0 . In fact, the tuning metric to evaluate neuronal participation in kinematics should depend not only upon the mean firing rate in a certain direction, but also on the distribution of the neural spike patterns. Normalizing by only the firing rate may not be the best way to evaluate neuron tuning. We propose an information theoretic tuning depth

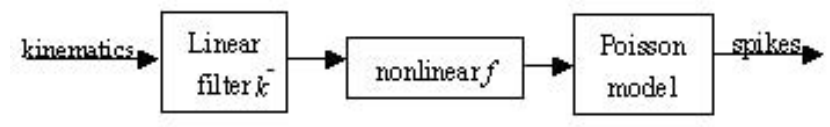

Fig. 1. Block diagram of projected-nonlinear-Poisson model

as a new metric for evaluating neuron receptive properties between neural activity and animal movement's directions. Consider that a tuned cell is defined as a cell that we expect to extract more information between the neural spiking and the angle direction. If a cell is tuned to a certain angle, the well-established concept of mutual information [16], can mathematically account for an information theoretic metric between the neural spikes and direction angles, which is given by:

$$
I(s p k ; \theta)=\sum_{\theta} p(\theta) \sum_{s p k=0,1} \log _{2}\left(\frac{p(s p k \mid \theta)}{p(s p k)}\right)
$$

where is the probabilistic density of all the direction angles, which can be easily estimated by Parzen window [17]. The direction angles of the kinematic vectors are normalized to a value between $-\pi$ and $\pi$. The histogram of the direction is drawn and normalized to approximate $p(\theta)$. $p$ (spike) can be calculated simply as the percentage of the spike count during the entire spike train. $p(\operatorname{spk} \mid \theta)$ is the conditional probability density of the spike given the direction angle. For each neuron, the histogram of the spike-triggered angle is drawn and normalized to approximate . In other words, the direction angle is accounted for in the histogram during the corresponding direction angle bin only when there is a spike. Then $p(s p k=1 \mid \theta)$ is approximated by dividing the histogram of $p(s p k=1, \theta)$ by the histogram of $\theta$, which is in fact Bayes rule.

$$
p(\operatorname{spk}=1 \mid \theta)=\operatorname{fracp}(\operatorname{spk}=1, \theta) p(\theta)
$$

where $p(\operatorname{spk}=0 \mid \theta)=1-p(\operatorname{spk}=1 \mid \theta)$. When $p(\theta)$ is 0 , $p(s p k=1, \theta)$ is set to be 0 . Note that because $p(s p k, \theta)$ is always smaller than $p(\theta)$, this actually does not share the same problem as (2).

\section{Estimation of Causal Time Delay in Motor Cortex}

The information theoretic formulation can also be extended to estimate the causal time delay of a motor cortex neuron. Based on the projective nonlinear-Poisson model [9], shown in Fig 1, that encodes the neural response to stimulus, the motor cortical cell can be modeled as

$$
\begin{gathered}
\lambda_{t}=f\left(\vec{k} \cdot \vec{v}_{t+l a g}\right) \\
\text { spike }_{t}=\operatorname{Poisson}\left(\lambda_{t}\right)
\end{gathered}
$$

where $\lambda_{t}$ is the instantaneous firing rate in an inhomogeneous Poisson spike generator. $f$ is the nonlinear function, representing the nonlinear neural property. $\vec{k}$ is a linear filter, representing the preferred direction. $\vec{v}_{t+\text { lag }}$ is the velocity vector with causal time delay corresponding to spike . $_{\text {. }}$ 

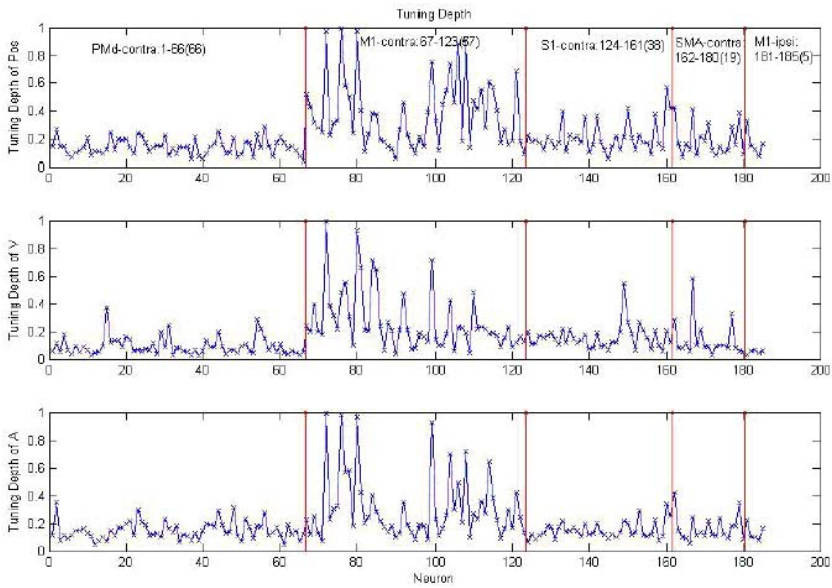

Fig. 2. Traditional tuning depth for all the neurons computed from three kinematics
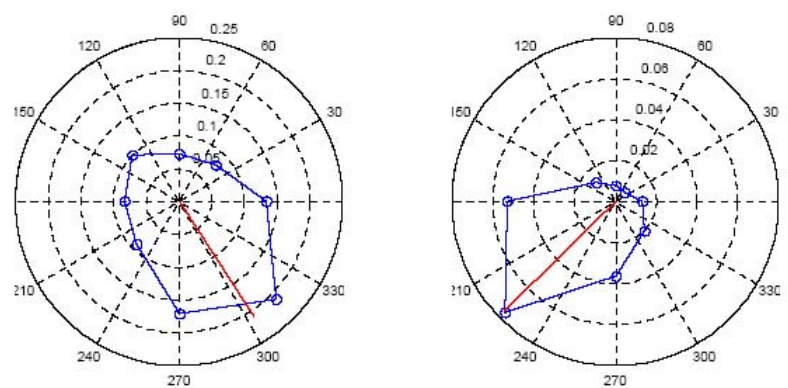

Fig. 3. A counterexample of neuron tuning evaluated by tuning depth

Similar to the evaluation of the information theoretical tuning depth, the mutual information between the spike and the delayed linear filter kinematics vector is first drawn simply as the function of the time lag after a spike as in (3).

$$
\begin{array}{r}
I_{(s p k ; \vec{k} \vec{x})}(\text { lag })=\sum_{\vec{k} \vec{x}} p(\vec{k} \vec{x}(\text { lag })) \sum_{s p k=0,1} p(s p k \mid \vec{k} \vec{x}(\text { lag })) \\
. \log _{2}\left(\frac{p(s p k \mid \theta)}{p(s p k)}\right)
\end{array}
$$

where the approximation to $p(s p k \mid \vec{k} \vec{x}($ lag $))$ is exactly the same as the estimation of $p(s p k=1 \mid \theta)$. The time delay with most mutual information is assigned as the best time lag for each neuron.

\section{RESULTS AND ANALYSIS}

For our BMI data, neural activity is characterized as binary spike trains. The traditional tuning depth for all 185 neurons computed from three kinematics variables of hand position, velocity and acceleration are shown in Fig 2. The top plot is tuning depth computed from position, the middle from velocities, and the bottom from accelerations. The cortical areas where the micro-arrays were placed are also marked in the figure. We can see clearly that most tuned neurons are in the primary motor cortex regardless of which kinematic vectors are used to calculate the tuning depth.
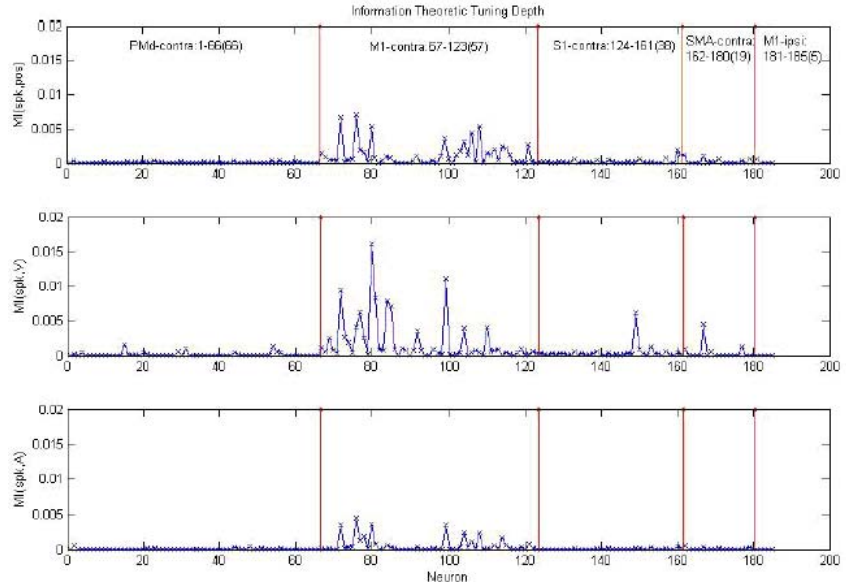

Fig. 4. Information theoretic tuning depth for all the neurons computed from 3 kinematics plotted individually
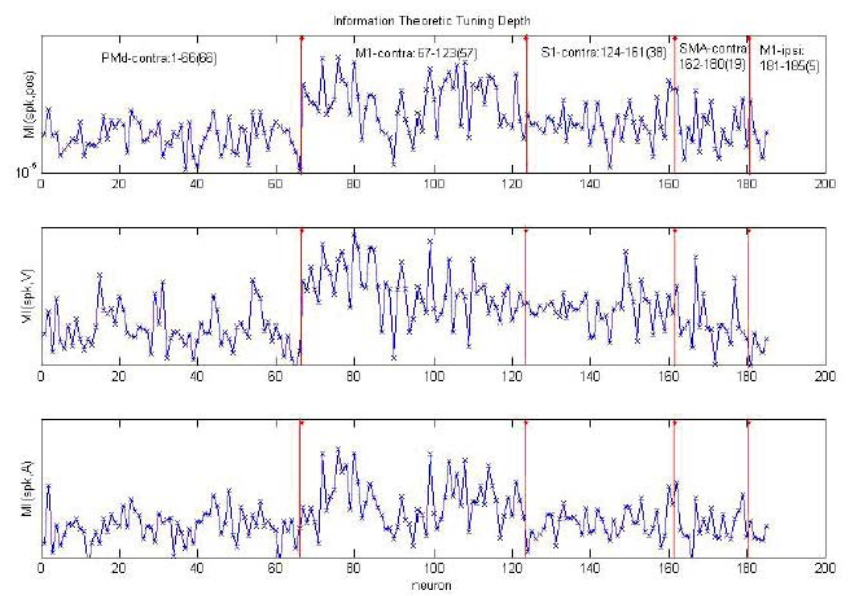

Fig. 5. Information theoretic tuning depth in logarithmic scale for all the neurons computed from 3 kinematics plotted individually.

Fig. 3 shows two neural tuning curves evaluated by tuning depth, where the red lines represented the preferred direction evaluated by (1). The left plot is a tuning plot of neuron 72 with tuning depth 1 . The right plot is for neuron 80 with tuning depth 0.93 . Neuron 72 fires less in other directions than the preferred one, while neuron 80 does not fire in any direction except the preferred one. By visually inspecting the plots, we can infer that neuron 80 is more "tuned" than neuron 72. However, using the tuning depth metric, neuron 80 was assigned smaller tuning depth.

Evaluated by the proposed metric, the information theoretical tuning depth gives $1.60 \mathrm{e}-2$ to neuron 80 , and $0.94 \mathrm{e}-$ 2 to neuron 72. Moreover, the new approach offers much more fine resolution between neurons with similar tuning allowing the quantification of subtle tuning differences. Fig. 4 shows the information theoretic depth calculated from all 3 kinematic directions for all the cells. Compared to Fig.2, in which the tuning depths are normalized to $[0,1]$ for all kinematics, the mutual information shows clearly that the velocities (the middle plot) conveys more tuning information than position or acceleration, as reported in the literature. 


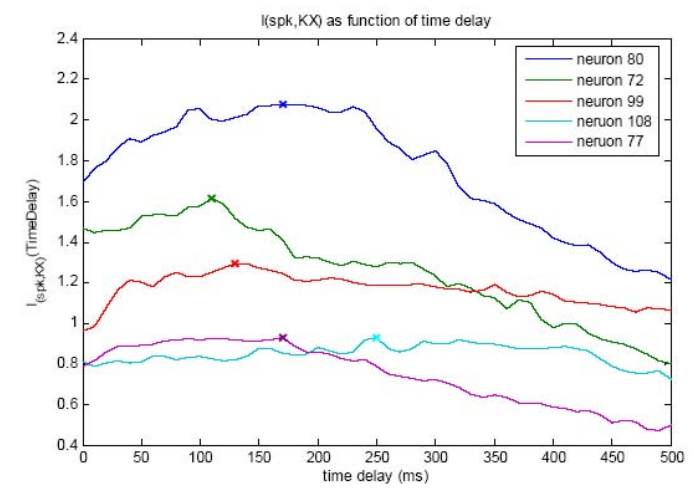

Fig. 6. Mutual information as function of time delay for 5 neurons

Since mutual information is a distance (it is self-normalized) it allows the relative assessment of tuning across different kinematics. For example, we found that neuron 121 is tuned more to position, while neuron 149 is tuned more to velocity. In Fig. 4, with the exception of the M1 cortical area, the neuronal information theoretic tuning depths seem almost flat, which could be erroneously interpreted as meaning that these neurons have little or no tuning. Actually, the mutual information is a nonlinear measure, emphasizing the large distances. Therefore, when displayed in logarithmic scale, the difference between neurons in other cortical area is made much clearer in Fig. 5.

In order to reduce the computational complexity of the BMI model, the 30 most tuned neurons could be selected as candidates to decode the movements. For this dataset, there are 3 neurons in PMd-contra, 21 neurons in M1-contra, 3 neurons in S1-contra, and 3 neurons in SMA-contra.

In order to estimate the causal time delay of these motor cortical neurons, we selected a possible delay range from 0 to $500 \mathrm{~ms}$ after a neuron spike. For all 185 neurons, the mutual information as a function of time delay was obtained from 10,000 continuous samples during movement. The time delay with highest mutual information was assigned as the best time lag for each neuron. Fig. 5 shows the mutual information as a function of time delay after spikes for 5 neurons: neuron 72 , neuron 77 , neuron 80 , neuron 99 , and neuron 108. The best time lags are marked by a cross on each curve, and are $110 \mathrm{~ms}, 170 \mathrm{~ms}, 170 \mathrm{~ms}, 130 \mathrm{~ms}$ and $250 \mathrm{~ms}$ respectively. It is interesting to observe that not all the neurons have the same time delay, although all of these neurons are in M1. The average best time delay for all 185 neurons was $220.108 \mathrm{~ms}$.

\section{CONCLUSION AND DISCUSSION}

Here we demonstrated that the traditional formulation for tuning depth has limited ability for interpreting the timing information in neuronal firing and its relation to kinematic variables. The information theoretic tuning depth proposed seems to provide a more detailed perspective because it statistically quantifies the amount of information between the kinematic vectors triggered off by the spike train. Moreover, since MI is a distance, it does not need a "hard" normalization between [0,1] allowing the comparison of tuning importance for different kinematics, and therefore can provide relative insight to the significance of each kinematic variable. By setting a single threshold, the metric can also help determine a subset of neurons most tuned to the task which would reduce the computational complexity of BMI modeling. In addition, the information theoretic formulation naturally leads to the estimation of the functional time delay of motor cortex neurons, which has not been systematically addressed. The nonlinear sensitivity of MI can be counteracted with a logarithmic plot for visual analysis.

\section{ACKNOWLEDGMENTS}

The authors would like to thank Dr. Miguel Nicolelis for the use of the monkey data collected in his laboratory at Duke University.

\section{REFERENCES}

[1] Georgopoulos A. P., Lurito J. T., Petrides M., Schwartz A. B., and Massey J. T., "Mental rotation of the neuronal population vector", Science, 1989, vol. 243, pp. 234-236

[2] Georgopoulos AP, Kalaska JF, Caminiti R, Massey JT, "On the relations between the direction of two-dimensional arm movements and cell discharge in primate motor cortex", J. Neurosci,. 1982, 2:1527-1537.

[3] Georgopoulos A. P., Schwartz A. B., and Kettner R. E., "Neuronal population coding of movement direction", Science, 1986, vol. 233, pp. 1416-1419

[4] Taylor D. M., Tillery S. I. H., and Schwartz A. B. , "Direct cortical control of 3D neuroprosthetic devices", Science, 2002, vol. 296, pp. $1829-1832$

[5] Sanchez J. C. , "From cortical neural spike trains to behavior: modeling and analysis", PhD. Dissertation, 2004, University of Florida

[6] Moran D. W., Schwartz A. B. , "Motor cortical representation of speed and direction during reaching," J. Neurophysiol., 1999, 82: 2676-2692

[7] Roitman A. V., Pasalar S., Johnson M. T. V., Ebner T. J. , "Position, direction of movement, and speed tuning to cerebellar purkinje cells during circular manual tracking in monkey", J. Neurosci., 2005, 25(40): 9244-9257

[8] Eden U. T., Frank L. M., Barbieri R., Solo V., Brown E. N., "Dynamic analysis of neural encoding by point process adaptive filtering", Neural Comput,. 2004, 16(5): 971-998

[9] Simoncelli E.P., Paninski L., Pillow J., Schwartz O., "Characterization of neural responses with stochastic stimuli", The New Cognitive Neurosci., 2004, 3rd edition, MIT Press

[10] Chichilnisky E. J., "A simple white noise analysis of neuronal light responses", Network: Comput. Neural Syst., 200112: 199-213

[11] Nicolelis, M.A.L., Ghazanfar, A.A., Faggin, B., Votaw, S., Oliveira, L.M.O. "Reconstructing the engram: simultaneous, multiple site, many single neuron recordings". Neuron 18, 1997, pp. 529-537

[12] Wessberg J, Stambaugh CR, Kralik JD, Beck PD, Laubach M, Chapin JK, Kim J, Biggs SJ, Srinivasan MA, Nicolelis MA. "Real-time prediction of hand trajectory by ensembles of cortical neurons in primates", Nature, 2000, 408:361-365

[13] Kim S. P., "Design and analysis of optimal encoding models for brain machine interfaces", PhD. Dissertation, 2005, University of Florida

[14] Carmena J. M., Lebedev M. A., Crist R. E., O’Doherty J. E., Santucci D. M., Dimitrov D. F., Patil P. G, Henriquez C. S., Nicolelis M. A. L., "Learning to control a brain machine interface for reaching and grasping by primates", PLoS Biology, 2003, vol. 1, issue 2, 193-208

[15] Jammalamadaka S. R. and SenGupta A., Topics in Circular Statistics. River Edge, NJ: World Scientific Publishing Company, 1999

[16] Fazlollah M. Reza. An Introduction to Information Theory. New York: McGraw-Hill 1961. New York: Dover 1994.

[17] Parzen E., On the estimation of a probability function and the mode, Annals of Mathematical Statistics, 1962, vol. 33, no. 14, pp. 10651076 